\title{
EL PROCESO DEL CAMBIO TECNOLÓGICO EN ZONAS DE BORDE. EL CASO ANDALUZ (ESPAÑ̃)*
}

\author{
Por \\ Rosa Jordá Borrell**
}

\begin{abstract}
RESUMEN
El tema central del artículo es abordar el proceso de cambio tecnológico de una zona perférica de borde (Andalucía) situada al sur de la Unión Europea y de España y, que además, hace frontera con Marruecos a través del estrecho de Gibraltar. Este proceso de cambio provocado, en nuestra opinión, por el mercado único y las instituciones regionales ha dado lugar entre 1990 y 1998 a una modernización tecnológica, a la conformación de la trama organizacional del entorno, a la aparición de algunos clusters innovadores relacionados con la industria que tiende a externalizar el proceso de producción (aeronáutica, automóvil) y al surgimiento de empresas innovadoras creadoras de investigación y desarrollo (I+D).
\end{abstract}

\begin{abstract}
The central therne of this article is a look at the process of technological change in a peripheral border zone (Andalusia) in the south of the European Union and Spain, and which, as well, borders Morocco across the Strait of Gibraltar. This process of change, in our opinnion, is due in part to the area's having only one market, and to the fact that between 1990 and 1998 the regional institutions have given place to a modernization technology. Other causes are the structure of the organizational scherne of the environment, the appearance of some innovative clusters related to the industry that tends to externalize the process of production (aeronautics, automobile) and the emergence of innovative, creative, research and development companies $(\mathrm{I}+\mathrm{D})$.
\end{abstract}

\section{INTRODUCCIÓN}

A la hora de abordar este tema conviene diferenciar entre los problemas de desarrollo de la innovación en zonas de frontera situadas en el interior de una zona desarrollada y por otra, aquellas otras emplazadas en áreas de transición entre países industrializados y periféricos que denominaremos

\footnotetext{
* Una versión preliminar de este artículo se presentó en el evento "Fronteras en perspectiva internacional" efectuado en la Universidad Autónoma de Baja California los días 17 y 18 de noviembre de 1997.

** Profesora titular en la Universidad de Sevilla.
} 
áreas de borde, en las cuales existe un gradiente de renta, riqueza y formación importante. Nosotros nos vamos a centrar en estas últimas, en las de borde, haciendo hincapié en la problemática del cambio tecnológico y de la introducción de la innovación, para ver de qué forma esas áreas son capaces de apropiarse del crecimiento que genera la innovación a partir de la adaptación y mejora de tecnología importada en el contexto de la globalización. Por otra parte, conviene aclarar que el término innovación se utiliza aquí en un sentido general y no en su acepción más restringida y específica relativa a la creación.

Evidentemente falta todavía profundizar más en cómo los países/regiones que no han seguido el esquema clásico pueden conseguir ventajas competitivas a partir de la innovación. Pero los resultados de algunos trabajos empíricos efectuados recientemente nos pueden arrojar luz sobre estas cuestiones. Así Porter (1994) demuestra que la diferencia entre las zonas de frontera de países desarrollados y las de borde radica en la fase en que se encuentra el proceso innovador. Por ejemplo, en las zonas de frontera europeas que no son periféricas, la característica más importante es la cooperación entre las empresas y los centros de investigación dado que el sistema ciencia-tecnología-industria está bastante maduro; las estructuras interfase juegan un papel importante en el apoyo de la innovación y de la transferencia de tecnología; los planes de estudios se hallan interrelacionados con las necesidades de las empresas locales (politécnicas como institutos de formación profesional) y la administración desempeña el papel de árbitro en la introducción del cambio tecnológico y de la innovación, dictando medidas para que empresarios y trabajadores se responsabilicen conjuntamente.

Otros trabajos realizados recientemente afirman que el análisis de la exportación tecnológica constituye un factor estratégico fundamental (Molero, 1966), a partir del cual se podría llegar a la situación adecuada para obtener ventajas competitivas mediante la adaptación y mejora de la tecnología importada, pero que en el caso de España iría ligado al desarrollo de sectores que hayan creado mercados oligopólicos, las empresas estén participadas por capital extranjero y, además éstas, hayan realizado un gran esfuerzo investigador. En cualquier caso, los expertos en el tema afirman que los países/regiones deben disponer de un alto índice de aprendizaje colectivo para obtener una tasa de crecimiento elevada mediante el desarrollo del proceso de la innovación (Lucas, 1993; Gaffard y Quéré, 1996).

Un tercer grupo de autores demuestran que el papel del mercado y de las instituciones (nacionales y regionales) ocupan un lugar privilegiado a la hora de explicar el cambio tecnológico (Dosi, 1992; Porter, 1994; Jordá 
Borrell, 1997) de las regiones borde a través de la implantación de infraestructura tecnológica y de la generación de entorno. Las implicaciones de la globalización en la búsqueda de nuevos productos y nuevas formas de organización de la producción, las políticas de los países desarrollados para detener la inmigración y el desarrollo de mercados regionales en contextos amplios (ALENA, mercado único, etcétera), han conducido a los grandes grupos industriales a establecerse en las zonas de borde mediante la incorporación de la automatización y de las nuevas tecnologías de organización flexible, incorporándose así dichos territorios a las fases iniciales de la innovación (generación de factores e inversión) de acuerdo con la metodología de Porter (1994). Aún así, como la conformación del sistema ciencia-tecnología-industria todavía se encuentra en un estadio de desarrollo embrionario, sobre todo por el bajo nivel tecnológico del tejido industrial autóctono, las instituciones deben poner en marcha una estrategia de innovación industrial y aplicar medidas capaces de movilizar recursos locales con poder de integración, interacción y organización.

Por ejemplo, Andalucía inició la etapa de creación y desarrollo de factores avanzados (las estructuras interfase y de servicios cuaternarios) en 1990, cuando empezó a hacerse patente la entrada de España en la Comunidad Europea (CE) y más tarde, con la puesta en marcha del mercado único. Estos hechos permitieron que la industria alcanzara importantes ventajas por medio de la estrategia de reducción de costes, al mismo tiempo que se realizaron fuertes inversiones en la modernización de los equipamientos, en la atracción de multinacionales y en la compra de licencias. Los resultados han sido que la tecnología avanzada se sitúa en pequeños reductos productivos, pero fundamentalmente el proceso innovador se desenvuelve en torno a la aplicación y mejora de los métodos y savoir faire extranjeros. Pues como afirma Porter (1994), para mejorar continuamente la productividad y aumentar la competitividad regional no se necesita exclusivamente desarrollar un proceso de creación de tecnología, basta con estimular la eficiencia de la producción, incrementar las características de los productos y elevar la calidad de éstos.

En nuestra investigación veremos también cómo la renovación de maquinaria y el proceso de externalización de la producción está modificando la organización de esta última y del trabajo en las empresas, e incluso ha creado nuevas cualificaciones, ha incrementado el nivel tecnológico de las Pequeñas y Medianas Empresas (a partir de aquí PYMES) y se han creado en ellas departamentos de ingeniería; sin embargo, únicamente han alcanzado el estadio de calidad un $30 \%$ de éstas, mientras que la diferenciación sigue siendo buscada fundamentalmente por las grandes empresas. 
Tampoco la introducción del diseño en las PYMES ha tenido demasiado éxito (programa del Centro de Diseño CEDIAN), programa de la Confederación de Empresarios Andaluces (CEA) del Centro de Difusión de las Nuevas Tecnologías de la Industria Textil) (Jordá Borrell, 1992) a pesar de la política de animación que se viene desarrollando desde 1990.

Por todo ello, cabe decir, de acuerdo con la metodología de Porter (1994), que nos encontramos en las fases iniciales a la innovación (generación de factores e inversión), en las cuales, la implantación del mercado único y el papel de las instituciones han servido para crear infraestructuras, servicios avanzados y para que los grandes grupos industriales externalicen parte del sistema productivo a las PYMES locales y hagan transferencia de tecnología a medida que estas últimas alcancen un nivel tecnológico adecuado.

También en la frontera norte de México ha habido un proceso generalizado y heterogéneo de adaptación de nuevas tecnologías debido a la atracción paulatina de multinacionales, localizadas allí estratégicamente para aprovechar las ventajas competitivas que se derivan de la política estadounidense, del proceso de globalización y de la entrada en vigor de ALENA. Contrariamente a la idea difundida en un principio de que el cambio tecnológico produciría el traslado de las plantas a sus lugares de origen (USA, Japón, países asiáticos, entre otros), la automatización y la organización flexible (industria electrónica, componentes, etcétera) han atraído nuevas empresas. De forma que encontramos cada vez operaciones más complejas que readaptan las actitudes de los operarios, asumiendo éstos mayores responsabilidades, en línea con lo que sería un modelo altamente flexible de organización del trabajo. Esto es esencialmente relevante en el caso de las nuevas maquiladoras, pero algunas de las antiguas, en particular las de autopartes tampoco han quedado al margen de esta tendencia.

Así pues, el desarrollo tecnológico de la frontera norte de México está enfocado a hacer pequeñas mejoras y adaptaciones a los procesos productivos. Las empresas maquiladoras han introducido los nuevos sistemas de calidad y empiezan a mantener una vinculación más estrecha con el sector educativo. Pero los planes de estudio todavía no se hallan interrelacionados con las necesidades de las empresas locales. Por otra parte, las áreas de oportunidad para desarrollar tecnología aún no aportan herramientas para contribuir al desarrollo del resto de los sectores económicos. Diríamos que el estado de Baja California (México) se encuentra en la fase de creación de factores dentro del proceso de innovación.

De ahí que los objetivos de nuestra investigación vayan encaminados a demostrar, a través del ejemplo andaluz, cómo las zonas de frontera de 
borde pueden ser capaces de superar los problemas que plantea la puesta en marcha de un proceso de innovación a partir de un sistema ciencia-tecnología-industria (SCTI) inmaduro. Para ello nos vamos a centrar primero, en cómo la renovación de maquinaria está incidiendo en la reducción de costes y en la diferenciación del producto y, a su vez, está modificando la organización del trabajo en las empresas, si bien previamente vamos a explicar de qué manera se han configurado las etapas de conformación del SCTIA. En segundo lugar, se analizarán las implicaciones del mercado único en la búsqueda de nuevos productos y nuevas formas de organización de la producción por parte de los grandes grupos industriales para iniciar el proceso de creación de tecnología.

El tercer objetivo consiste en analizar la incidencia del entorno en el cambio tecnológico andaluz, midiendo el papel de las externalidades y de los efectos de desparramamiento en la creación de economías de escala y de aprendizaje. Para ello, como en el punto anterior, habremos estudiado el proceso de descentralización productiva provocado por la entrada en vigor del mercado único, haremos hincapié en las empresas innovadoras capaces de crear tecnología.

En este trabajo vamos a ofrecer también un panorama aproximativo del tamaño del aparato organizacional que se ha desarrollado en Andalucía con el propósito de inferir si la dimensión alcanzada es la adecuada para el mercado existente; así como trataremos de dar una visión de conjunto de los impactos producidos por los efectos de desparramamiento, explicar la intensidad de los flujos producidos por la cooperación y analizar la posibilidad de desarrollo de las economías de escala y de aprendizaje resultantes.

\section{ETAPAS DE CONFORMACIÓN DEL PROCESO INNOVADOR}

Desde los primeros años de la institucionalización de la autonomía andaluza (1978) la política dirigida a transformar la estructura productiva ha sido incierta, produciéndose cambios de énfasis significativos tanto en las prioridades sectoriales como en el carácter endógeno o extrovertido del desarrollo, así como en el protagonismo del sector público debido a la problemática económica y social andaluza.

Esta situación difícil de superar dio lugar a que la Junta de Andalucía se haya visto obligada, no sólo a cambiar la orientación política del proceso de innovación, sino también a diseñar el modelo de forma escalonada en el tiempo, en función de la capacidad transformadora de la economía, de la apertura exterior del sistema y de las características del sector empresarial. Por ello, la pauta que hasta ahora ha seguido la Junta de Andalucía 
ha sido generar primero el marco político y normativo para cada etapa y después, crear las estructuras que tenían que poner en marcha los instrumentos. Aún así, el proceso se ha desarrollado de forma discontinua en el tiempo, pudiendo situar el comienzo del proceso de innovación en torno a finales de la década de los ochenta, cuando la entrada en la Unión Europea se deja sentir en Andalucía. De ahí que las fases del proceso se superpongan paralelamente debido a que el sistema no es capaz de conseguir resultados en los plazos adecuados, ni de difundir el proceso de innovación en los sectores económicos y en el espacio de forma homogénea.

Así pues, cabría diferenciar en el proceso de innovación andaluz tres etapas superpuestas, conformadoras de la base de acumulación que el SCTIA necesita desarrollar para propiciar el cambio tecnológico:

La primera se inicia a comienzos de los años ochenta y viene marcada por el establecimiento del marco legal para generar tejido industrial a partir de la ley de incentivos regionales, así como por la publicación del Programa de política científica en el marco del Plan económico andaluz 1984-1986, cuando la Junta, en 1984, asume las competencias en materia de investigación y desarrollo (I+D). Si bien, todavía durante estos años la política regional no tiene como tema prioritario el desarrollo dei proceso de innovación debido a las dificultades económicas y a la falta de cultura técnica.

La segunda fase se inicia en 1990 y viene enmarcada por el giro que da la política económica andaluza con motivo de la entrada de España en la Comunidad Europea. Durante este periodo se crea el I Plan Andaluz de Investigación (PAI) y se decide apoyar plenamente en el presupuesto del Plan Andaluz de Desarrollo Económico (PADE) 1991-1994 el desarrollo de $\mathrm{I}+\mathrm{D}$, la implantación de infraestructura tecnológica y la creación del tejido empresarial. Unos años más tarde, cuando se publica el Plan Industrial 1994-1997 se pone en marcha la política de modernización empresarial y de generación de entorno. Pero, a pesar de que el marco político normativo hecho público se había enfocado también hacia el entorno productivo, el gobierno regional decidió apoyar fundamentalmente al subsistema científico, sin poner en marcha una estrategia clara de innovación industrial debido a las características de subdesarrollo que presentaba el tejido industrial autóctono y a la situación embrionaria del sistema ciencia-tecnología-industria.

Apoyándose en las dos fases anteriores, creemos que la Junta de Andalucía puso en marcha en 1996 (Jordá Borrell, 1997) la tercera fase del proceso de innovación, cuando creó el Plan Andaluz de Investigación II, en el cual se publican las medidas necesarias para articular el subsistema productivo con el científico y fomentar la apertura al exterior, mediante 
instrumentos de fomento a la interacción y adoptando como pilares los parques tecnológicos y el Centro de Enlace del Sur de Europa (CESEAND).

En esta etapa se está introduciendo un cambio fundamental en el proceso de innovación centrado en el mayor interés mostrado por las instituciones regionales por apoyar a la PYME tanto en los parques tecnológicos como en el conjunto del tejido industrial. Posiblemente porque se han dado cuenta de la importancia de transformar los entornos locales mediante el desarrollo de la difusión y la cooperación a fin de articular a la economía andaluza en el proceso de globalización mediante sistemas de organización en redes. Para ello, las instituciones regionales y las infraestructuras interfase tendrían que conocer más en profundidad las necesidades de las empresas, sobre todo de las PYMES, y darles mayor participación en la gestación del proceso de innovación.

Pero, hasta ahora, únicamente se han realizado algunos estudios sobre las necesidades de las PYMES, por ejemplo, se ha llevado a cabo el Informe RTTTS $^{1}$ (1996), se han tomado medidas para interrelacionar a través del CESEAND las estructuras puente más significativas y se ha hecho una primera evaluación de la oferta de servicios de apoyo a la innovación y a la transferencia tecnológica. Sin embargo, aún no se ha puesto en marcha una política de innovación eficaz y potente, capaz de transformar los entornos locales mediante el desarrollo de la difusión y la cooperación que den lugar a un nuevo sistema de organización en redes.

Paralelamente a estas etapas, nosotros formulamos la hipótesis, tal como hemos dicho antes, que se están empezando a desarrollar otras dos más, de forma espontánea y casi paralela que tienen como protagonistas a las multinacionales y grandes empresas que han adoptado el proceso de descentralización productiva con motivo de la entrada en vigor del mercado único. Estas etapas se pueden considerar representativas del cambio tecnológico que ha dado comienzo en torno a 1993, a pesar de que la Consejería de Economía y Hacienda puso en marcha los incentivos de modernización tecnológica en 1984.

Así, la primera fase vendría definida por la implantación en algunos sectores (agroalimentario, metalmecánico, eléctrico, electrónico e informático, minas y energía, químico y textil y plásticos) de la automatización y las nuevas tecnologías de organización de la producción, de gestión y del trabajo para alcanzar la competitividad vía reducción de costes. Y, la segunda, aunque se está introduciendo de una forma muy tímida, estaría

\footnotetext{
1 Regional Innovation and Technology Transfer System, proyecto de investigación de la Unión Europea que se aplicó en Andalucía entre 1994-1996.
} 
representada por el paso de la empresa andaluza hacia la competitividad mediante el incremento de la calidad del producto y la diferenciación. Fases que estarán situadas en el proceso de innovación en un estadio inferior (salvo excepciones), el que se requeriría alcanzar para que li generalidad de las empresas realicen recursos tecnologicos propios, pero que al conllevar cambios organizativos e implicar procesos de aprendizajes de distinto tipo pueden entrañar la formación de economías de escala externas y el aprovechamiento de efectos de desparramamiento.

\section{Análisis de impactos}

Los estudios que hemos realizado (según una encuesta llevada a cabo a 100 empresas a lo largo de 1996-1997 y de las consultas efectuadas a las bases Sibila y Sie del Instituto de la Pequeña y Mediana Empresa (IMPI) demuestran que la gran mayoría de los empresarios encuestados incorporaron la automatización y las nuevas tecnologías de organización de la producción de forma paralela. Pero más de $39 \%$ añadió que la introducción de equipamientos automáticos constituye el motivo principal de estímulo para encontrar nuevas respuestas organizativas a su proceso productivo.

Precisamente, esa búsqueda por conseguir una mejor organización de la producción les llevó a realizar trabajos de ingeniería para adaptar los nuevos equipos a sus necesidades, realizando alteraciones de las normas técnicas correspondientes a la nueva maquinaria adquirida. Así, de las 100 empresas entrevistadas, $62 \%$ tiene personal trabajando en un departamento de ingeniería, aunque la mayoría de éstos no llegan a los 8 empleados. Por otro lado, $13 \%$ emplea más de 35 personas para esta actividad.

La combinación de las dos propiedades incorporadas al nuevo proceso productivo (integración y flexibilidad) permitió, por una parte, obtener altos rendimientos de la mano de obra y del capital fijo y por otra, una transformación de la organización de la producción y del trabajo a partir de la descentralización de las actividades y de las responsabilidades, aumentando la producción y diversificándola e incrementando la autonomía del método de trabajo; en consecuencia, los empresarios descubrieron la necesidad de aumentar la polivalencia de los trabajadores (cuadro 1). Además, la flexibilidad de los equipos produjo una mejor utilización de las capacidades instaladas, un poder de reaccion inmediata a las variaciones del mercado y una mejora en el tiempo de vida de operación del equipamiento, en la medida en que se puede pasar fácilmente de fabricar un producto a otro sin que sea necesario sustituir la máquina. 


\section{CUADRO 1.}

\section{Cambios en la organización del trabajo}

Aumento de la polivalencia de los trabajadores

Incremento de los requerimientos de especialización

Autonomía del método de trabajo

Mayor control de la dirección sobre el trabajador

Mayor seguridad

Mejores condiciones de trabajo.

\section{Cambios en el trabajo}

Reducción del nivel de empleo en la industria

Aumento de las exigencias de cualificación y reciclaje

Áreas de la empresa donde se redujo el nivel de empleo

Administración

Fabricación y movimiento de materiales

Fuente: A partir de nuestra encuesta (1996).

Si diferenciamos las empresas por tamaños, el cambio tecnológico en las PYMES ha servido para elevar el nivel tecnológico y para disminuir los stocks intermedios, los tiempos medios, las pérdidas de materiales, factores todos que han favorecido la introducción de la competitividad a través de la reducción de costes, el incremento de la producción, la flexibilidad y el aumento del nivel tecnológico y, además, les han llevado a experimentar la necesidad de acceder a la formación para aplicar y utilizar los nuevos equipos.

Sin embargo, en las grandes empresas al introducir la automatización implantaron las nuevas tecnologías de gestión y de organización, haciendo más hincapié en estas últimas. De ahí que los cambios fueron mucho más impactantes en la elevación de la calidad del producto y en la búsqueda de diferenciación que en el incremento del nivel tecnológico. Así, según la base de datos Sibila y Sie, una cuarta parte de las empresas innovadoras llevaron a cabo modificaciones sustanciales de los productos ya existentes, lo que nos lleva a corroborar que estas empresas han adoptado la estrategia tecnológica a partir de la mejora de la calidad y de la diferenciación. A su vez, la adopción de esta estrategia está generando la necesidad, por un lado, de introducir los sistemas de control de calidad (total o círculos de control de calidad), las técnicas de just in time, sistemas de control de producción, sistemas de planificación y control de inventarios, lo que ha dado lugar a 
un incremento sustancial de la productividad. Y, por otro, la búsqueda de diferenciación llevó en muchos casos a la implantación de equipamientos especiales para fabricar lotes pequeños.

En resumen, la incorporación de la automatización y de las nuevas tecnologías de organización flexible ha supuesto un $57.6 \%$ de la inversión en nuevos equipos entre 1994 y 1996 en las empresas entrevistadas, y lo que es más importante aún, significa la afloración en las grandes empresas de la necesidad de presionar a las PYMES para que incorporen equipos automáticos y flexibles. Pero, como el cambio tecnológico tal como se está realizando, implica un proceso largo (debido a los problemas estructurales de la empresa andaluza) y las exigencias de descentralización de los nuevos sistemas de producción flexible requieren desarrollarlo rápidamente (entrada en vigor del mercado único), la Junta ha contemplado la posibilidad de diseñar planes especiales para las PYMES que formaban parte de los sectores prioritarios, aunque hasta el momento sólo se ha aplicado al sector metalmecánico.

En segundo lugar, la combinación de búsqueda de diferenciación, la introducción de la calidad y la mejora tecnológica de los productos ha llevado en algunos casos a las empresas andaluzas a desarrollar investigación y desarrollo tecnológico (I+DT), y a implantar departamentos específicos, aunque muchas veces sin dotación de personal, tal como después veremos. Por ello, la segunda fase del proceso de innovación vendría introducida por el paso de la empresa hacia la competitividad tecnológica mediante el incremento de la calidad del producto y de la diferenciación. De forma que, con la expansión de esta etapa, si llega a realizarse, el tejido productivo andaluz habría desarrollado ya un sustrato tecnológico suficientemente complejo para acometer las actividades de I+D de manera generalizada.

Una vez explicados los impactos producidos en la organización de la producción en las empresas, nos vamos a centrar en la capacidad del mercado en crear cualificaciones diferentes a pesar de que el proceso de innovación todavía no es capaz de modificar las características de la economía ni de transformar la estructura de la producción, puesto que el compromiso de las empresas con la innovación todavía depende mucho de los niveles y cambios en los precios relativos que de las oportunidades tecnológicas. Y, por otra parte, a dicho proceso de innovación aún le queda mucho camino por recorrer para que llegue a ser multilocal, multisectorial y multifuncional, pudiéndolo calificar en estos momentos de desequilibrado, por su fuerte polarización territorial (localizado sobre todo en torno al área metropolitana de Sevilla, Málaga, Bahía de Cádiz y puntualmente en Jaén) y sectorial. 
Así, de acuerdo con nuestra encuesta, las empresas que han incorporado las nuevas tecnologías de organización de la producción y de la gestión han reducido el número de trabajadores en las áreas de administración, fabricación y movimiento de materiales. Pero sin embargo, están demandando, por un lado, un mayor nivel de preparación y de reciclaje de la mano de obra basado en la polivalencia y en la especialización debido a las posibilidades de cambio ganadas con la flexibilidad y la integración del proceso productivo (cuadro 1). Y por otro, requieren un mayor número de personal con cualificaciones intermedias que tengan conocimientos informáticos, estadísticos, de idiomas, ingeniería. De forma que, el perfil del nuevo trabajador solicitado tendría que ser capaz de tener una visión global del nuevo proceso de fabricación, de mantenerlo y de adaptarse a los cambios de la empresa. Además, debe reunir cualidades que giren en torno a la responsabilidad, capacidad para trabajar en equipo, raciocinio lógico, iniciativa, etcétera. No obstante, los empresarios aducen que han tenido dificultades para incorporar mano de obra preparada debido a la necesidad de un cambio de valores y actitudes de los trabajadores, así como a los problemas que ofrece la reglamentación laboral.

En definitiva, aunque Andalucía se aleja de la diversificación tecnológica que caracteriza a la especialización de las economías centrales, se encuentra en el camino factible para llevar a cabo la modernización tecnológica en el subsistema productivo a través: 1) Del incremento del número de PYMES que se conviertan en industria auxiliar de las grandes empresas conforme se vaya expandiendo el proceso de innovación y Andalucía sea capaz de atraer más multinacionales. 2) De la transformación de los entornos locales mediante el desarrollo de la difusión y la cooperación. 3) Mediante los éxitos cosechados por la política económica andaluza en relación con la tasa de crecimiento de la demanda por la mejora del nivel de internacionalización de la empresa andaluza, lo que puede dar lugar a que se conserve el nivel de empleo actual y la pirámide ocupacional adopte la forma de rombo por el aumento de la demanda de cualificaciones intermedias.

\section{IMPORTANCIA DEL ENTORNO EN EL CAMBIO TECNOLÓGICO}

El estudio del entorno adquiere en Andalucía una importancia estratégica para asentar el cambio tecnológico, tanto a partir de la externalización del proceso productivo y la mejora continua de la eficiencia productiva como mediante la elevación de la calidad de los productos, por el desarrollo de 1+D. Las aportaciones de Porter y de la corriente evolucionista sobre la importancia de las políticas nacionales y/o regionales en la creación de 
entorno y en las fuentes de ventaja competitiva nos van a servii para plantear la relevancia de las economías de escala y de aprendizaje en función de la capacidad regional de generar externalidades y de saber aprovechar los efectos de desparramamiento de las innovaciones.

Por otra parte, la discusión teórica que han ido generando los investi gadores de la innovación durante la pasada década, especialmente os evolucionistas, ha supuesto la posibilidad de ampliar el estudio del entorno más allá del mercado, profundizando en el papel que desempeñan las instituciones en la creación de infraestructuras y de servicios avanzados para fomentar la transferencia y difusión de conocimientos nuevos y económicamente útiles.

De ahí, la importancia que se le concede al entramado organizacion:al de las infraestructuras y servicios avanzados y al análisis de los flujos y dc impactos, y en definitiva, a los estudios de evaluación del medio local, al objeto de medir si la empresa juega el papel de protagonista en las diferentes interacciones (a escala regional, nacional e internacional) qu relacionan la ciencia y la tecnología y en cada una de las etapas del procesu de innovación. Todo ello depende de que los subsistemas científico y tecnológico (centros de investigación aplicada, centros de innovación y transferencia tecnológica (I+TT), empresas de ingeniería y bienes dc equipo) hayan alcanzado el nivel adecuado de desarrollo. Y las instituciones hayan jugado un papel importante de animación a la cooperación y a la transferencia de conocimientos, y por último, se haya creado el tamaño de aparato organizacional adecuado al mercado.

Así pues, nosotros en esta segunda parte del artículo vamos ha investigar: a) si la proliferación de infraestructuras interfase y de servicios tecnológicos de carácter público han generado un entramado organizacional capaz de presentar una oferta ajustada al mercado existente; b) si la cooperación, la transferencia de tecnología y la subcontratación ligada a la incorporación de las nuevas tecnologías de organización flexible han conducido a crear economías de aprendizaje y efectos de desbordamiento. Para ello, hemos entrevistado a 20 estructuras interfase y hemos utilizado la encuesta efectuada a 100 empresas llevada a cabo entre 1996-1997.

\section{Papel de las externalidades en el desarrollo de $\mathrm{I}+\mathrm{DT}$}

Efectivamente, el rendimiento de una firma no sólo depende de los esfuerzos tecnológicos propios sino también de los conocimientos a los que se tenga acceso vía entorno local y desde el exterior. Pues, las empresas para generar tecnología necesitan por una parte, desarrollar canales de información, formando parte del nuevo proceso de organización en redes 
que se está dando a nivel mundial. Y por otra, que las vías de comunicación por donde circula el conocimiento tácito y codificado, a escala regional y local, sean eficaces y hayan alcanzado el nivel de madurez adecuado al tamaño del mercado existente; dado que el rendimiento individual de cada empresa dependerá de la magnitud y diversidad de las acciones tecnológicas de las otras unidades empresariales y del número y calidad de los centros de servicios y de investigación, sobre todo de aquellos que la efectúan de forma aplicada.

En Andalucía, el modelo interactivo innovador todavía se está generando, dado que la cooperación entre los distintos entornos (científico, tecnológico, financiero y productivo) y en el interior de los mismos es muy exigua (Jordá Borrell, 1997). A decir verdad, el entorno científico es el único que reúne condiciones para fomentar la cooperación con los subsistemas productivo y tecnológico. En efecto, este entorno ha alcanzado la madurez en la tasa de doctores por cada 1.000 habitantes sobrepasando la media española (2.2) y en el peso conseguido por cada área prioritaria (20\%) en el gasto de I+D nacional al superar la importancia que tiene la Comunidad Autónoma en población y en valor añadido bruto (VAB) respecto al conjunto español. Por otra parte, dicho subsistema ha llegado a ser competitivo en algunos campos en el ámbito internacional, fundamentalmente en las áreas prioritarias del Plan de Desarrollo Económico Andaluz (Jordá Borrell, 1997). Así, el gasto total de investigación realizado entre 1990-1995, en agroindustria, ciencias de la vida, medio ambiente, tecnologías de la producción y de la información representó $14.85 \%$ del presupuesto del PADE (1991-1994) dedicado al I+D. Por otra parte, el número de contratos del subsistema científico financiados por el Programa Marco, por el Plan Nacional de I+D y por la Administración Regional suman el 82\% del total de proyectos del SCTIA en 1996 y engloban al $50 \%$ de los equipos del Plan Andaluz de Investigación de 1992.

Sin embargo, uno de los problemas más relevantes que se les plantea a los planificadores del sistema SCTIA es cómo conectar los subsistemas científico y productivo, dado que el primero: No ha creado, hasta ahora, fuertes lazos con los medios locales, todo lo contrario, se trata de un subsistema extrovertido que tiende a relacionarse sobre todo con otros centros científicos extranjeros y/o nacionales. Así, el peso de las aportaciones financieras de las empresas locales para la investigación en el presupuesto universitario representan el $20 \%$ del presupuesto total investigador de 1994, sumando un total de 203 contratos auditados por la OTRIs (Oficina de Transferencia de Tecnologías) andaluzas (Jordá Borrell, 1997). Y, en la actualidad, sólo un cuarto de los equipos del Plan Andaluz de Investigación efectúan investigación directamente relacionada con el sector 
productivo, funcionando únicamente 21 redes conformadas para la realización de proyectos financiados por el Programa Marco, en el que colaboran empresas y el entorno científico, pertenecientes a las áreas de agroindustria, ciencias de la vida, tecnologías de la información, tecnologías de la producción y medio ambiente.

Lo que nos está indicando que en el entorno científico falta tradición en el establecimiento de relaciones con el tejido productivo que no ha sido superada con la puesta en marcha de las estructuras interfaz porque:

1) El I+D de la universidad y de los centros del CSIC (Centro Superior de Investigaciones Científicas) gira fundamentalmente hacia la investigación básica y los institutos de investigación aplicada puestos en marcha por la Administración regional y aún no han alcanzado el nivel adecuado de desarrollo para el mercado existente.

2) La universidad y los centros del CSIC todavía no ven claro la necesidad de reformar su gestión y su política de transferencia de tecnología, invitando a las empresas a participar activamente en sus respectivos consejos sociales.

3) Las condiciones de acceso de las empresas al I+DT de los centros de investigación no son las adecuadas para despertar en las empresas la necesidad de generar capacidad de absorción de conocimientos a fin de aprovecharse de la cultura técnica existente a nivel local e incrementar su propia productividad, porque:

a) Las áreas prioritarias del Plan Andaluz de Investigación se corresponden en el terreno productivo, (salvo las tecnologías de producción, de la información, medio ambiente y biotecnología) con los sectores tradicionales (poco evolucionados desde el punto de vista tecnológico) y con aquellos subsectores que no tienen un peso importante en la estructura económica andaluza, excepto la agroindustria; y aún así, el contenido de los programas de los centros de investigación, de las universidades y del CSIC (Centro Superior de Investigaciones Científicas) muestran una evidente desconexión con las necesidades planteadas por la empresa y fundamentalmente por la PYME, como después veremos.

b) Los empresarios tan sólo realizan $12 \%$ del $1+\mathrm{D}$ andaluz, solicitan un 0.90 de patentes al año y únicamente existen alrededor de 1.000 firmas verdaderamente innovadoras pertenecientes a los sectores químico, construcción de maquinaria, material electroelectrónico, informática, minería, agroalimentaria, metalúrgica y textil. Y de ese total de firmas, el CDTI (Centro para el Desarrollo Tecnológico Industrial) financió proyectos de investigación a 160 entre 1992 y 1995; y el Programa Marco (1990-1994) a 15 de ellas. Por lo tanto, dado el nivel tecnológico de la industria y las características de la infraestructura de investigación, esta última no puede 
ser aprovechada por las empresas, salvo la industria química que requiere desarrollo de investigación básica y aquellas firmas capaces de demandar investigación aplicada a la universidad y a los centros del csic.

De esta forma, dado que la diversidad sectorial y sobre todo la intraindustrial es muy escasa en el terreno innovador, para que las empresas puedan con el tiempo beneficiarse de los efectos de desbordamiento y alcanzar una situación de madurez en el ámbito de las economías de escala externas y de aprendizaje, se requerirá mejorar el sistema de información tecnológica y desarrollar durante un largo periodo de tiempo la cultura de cluster y la de asociacionismo para conseguir que el proceso de transferencia de tecnología se realice de forma más diversificada (consultoras, proveedores, centros tecnológicos, universidades, centros de investigación aplicada) y no exclusivamente con el cliente.

Además, las estructuras puente deberán emprender acciones comunes de colaboración para reforzar la interrelación sectorial desde el punto de vista de la transferencia tecnológica ofertando una infraestructura soporte adecuada a las PYMEs, fundamentalmente formación. Pues, conviene resaltar que no estamos hablando de sectores innovadores sino de algunas empresas pertenecientes a determinadas ramas industriales que desarrollan I+DT de forma aislada o cooperando en ciertas ocasiones con la Universidad, los centros del cSic y con otros agentes extranjeros. Así, de acuerdo con nuestra encuesta, en el único sector donde se está dando un aprovechamiento importante de las economías de escala mediante la cooperación en I+DT (para el desarrollo de componentes) es en las PYMES de la industria de la automoción.

Cuando nos preguntamos acerca de la frecuencia con que se lleva a cabo la tarea de I+DT, observamos que si bien estas empresas invierten entre $2 \%$ y $7 \%$ del valor de la facturación, $60 \%$ de ellas declararon en nuestras encuestas no tener trabajadores destinados a esa función. Lo cual indica que la dedicación al desarrollo de I+DT es esporádica. El $40 \%$ tienen alguna persona que desempeñe tareas de I+DT. Y de las que realizan algún tipo de acción, (química, energético, medioambiental, electroelectrónico y metalmecánico, etcétera) más de la mitad destinan menos de 3 empleados a estas labores, por lo que en la mayoría de casos no existe un departamento específico. Sin embargo, la media española se sitúa entre 3 y 5 personas (Jordá Borrell, 1997).

Por otra parte, según la información que disponemos, sólo las empresas que están ligadas al Parque Tecnológico de Málaga (PTA), a CASA (industria aeronáutica) y a ciertas empresas de tecnologías de la información (Alcatel, Fujitsu, Isofotón) (Jordá Borrell, 1992) han generado una estructura de relaciones formales para crear mercados estables de transferencia 
de tecnología a través de redes de cooperación tecnológica. Así, en el PTA existen 6 redes internas; 2 de ámbito local y regional y 7 internacionales. $\mathrm{Y}$ entre las industrias auxiliares de CASA un $20 \%$ han firmado acuerdos de cooperación para llevar a cabo I+DT conjuntamente y recibir transferencia de tecnología de multinacionales. Por ejemplo, el grupo ISA (asociación de empresas autóctonas de la industria aeronáutica) consiguió entre 19921993 que la McDonell Douglas pusiera en marcha un programa de difusión de tecnología con total independencia de CASA.

Por lo tanto, las firmas innovadoras, a pesar de que forman parte de ellas multinacionales y grandes empresas (CASA, Fujitsu, Alcatel, Abengoa, etcétera) y que además, tienen una edad media entorno a los 25 años de antigüedad, de las cuales $80 \%$ ya se encontraban en Andalucía antes de 1980 y $19 \%$ de ellas fueron creadas entre 1980 y 1993 no han sabido organizar en su entorno una malla de redes de cooperación de I+DT entre las industrias auxiliares, empresas suministradoras, firmas clientes capaces de desarrollar economías de escala externas importantes, que puedan producir un efecto de desparramamiento de las actividades innovadoras sobre el tejido industrial andaluz y transformar así la economía, salvo, en el caso de las PYMES de la industria aeronáutica donde se da una notable cooperación intersectorial. Pero aún así, algunas de ellas presentan problemas de falta de imagen tecnológica en el mercado nacional y/o extranjero y no conocen bien los canales y los medios de transferencia tecnológica.

Lógicamente, el nivel de internacionalización de las empresas innovadoras es irrelevante, y aunque ciertas firmas realizan transferencia de tecnología a muchos países subdesarrollados, como por ejemplo Marruecos y Sudamérica (por ejemplo: Sainco, Abengoa, Isofotón), e incluso algunas de ellas son líderes en su campo (Abengoa), efectúan inversiones directas en el extranjero (Abengoa), o realizan subcontratación internacional (CASA), sin embargo, hasta ahora sólo $3 / 5$ partes de ellas llevan a cabo exportaciones y de escasa cuantía. Así, $79.4 \%$ de las empresas exportan hasta un $20 \%$ de su volumen de ventas y $93 \%$ de ellas dedican al comercio exterior únicamente $9 \%$ de sus ventas. En este sentido cabe señalar que la insuficiencia puesta de manifiesto en la exportación de mercancías, guarda paralelismos importantes con las barreras existentes en la exportación de tecnología o con el escaso desarrollo de la inversión en el exterior, aunque en este momento no podamos ofrecer todavía datos al respecto.

Ante esta situación, la evolución del proceso de aprendizaje colectivo guarda relación con las características de la estructura competitiva de los sectores industriales en Andalucía (tamaño de las barreras de entrada para los nuevos competidores, poder de negociación de los proveedores y 
compradores y riesgo de aparición de productos sustitutivos) y con la articulación de las empresas andaluzas en la economía global y del mercado único. A estos factores, cabe sumar las dificultades, según los resultados de nuestra encuesta, de las empresas para llevar a cabo el cambio tecnológico a partir del desarrollo de I+DT y del control de calidad total por falta de formación en un $43 \%$. Los impedimentos de las firmas a la incorporación de las nuevas técnicas de la organización de la producción, del diseño, de la ingeniería de producción, por problemas de asesoramiento e información en un $20 \%$. O las barreras que deben salvar un $37 \%$ de ellas para mejorar la eficiencia de la producción mediante la incorporación en los procesos productivos de los métodos de learning by doing y learning by using a fin de mejorar sus productos.

Obstáculos que van unidos a la escasa existencia de redes de colaboración en el sistema ciencia-tecnología industria, (SCTIA), a la extraordinaria juventud de los nuevos institutos de investigación aplicada y de las asociaciones industriales en materia de $\mathrm{I}+\mathrm{TT}$, dando lugar a un mercado tecnológico estrecho y poco maduro donde el tejido empresarial no se encuentra preparado para aprovechar los llamados "efectos de desbordamiento (spillovers)" derivados de la cooperación y de la transferencia de tecnología llevados a cabo en el subsistema científico. Nos encontramos, pues, en una fase del proceso innovador en que los elementos del sistema productivo apenas tienen conexión con el exterior y con el resto de empresas desde el punto de vista de la I+TT y donde además, el proceso de difusión tecnológica en los sectores tradicionales prácticamente no tiene incidencia en las PYMES. Así pues, un escenario de estas características únicamente permitirá una circulación de información tecnológica y de acumulación de experiencia individual reducida, impidiendo la producción de economías de aprendizaje.

\section{Economías externas y modernización tecnológica}

Dadas las dificultades de la empresa andaluza por conseguir ventajas competitivas a partir de la creación de tecnología, pensamos que el desarrollo de externalidades va esencialmente ligado al doble proceso que supone la modernización tecnológica y la descentralización de la producción desde las grandes empresas a las PYMES con motivo de la puesta en marcha del mercado único y de la articulación de ciertas empresas en la economía global. Procesos que a su vez han dado lugar a iniciativas de Spin-off, al establecimiento de redes de subcontratación y a la consecución de acuerdos de colaboración de producción, marketing, distribución, asistencia al cliente: generados tanto en el interior del Parque Tecnológico de 
Málaga como en las industrias del automóvil, aeronáutica, electrónica, informática, energética y metalmecánica. Es decir, en aquellos sectores que destacan por albergar empresas innovadoras.

En efecto, la modernización tecnológica unida a la incorporación de las nuevas tecnologías de la información, organización y automatización, han permitido que en los últimos diez años 1310 empleados hayan dejado la empresa madre para crear la suya propia. De estos casos han surgido en el mismo periodo, 3406 proyectos empresariales que han supuesto la implantación de 4585 empleos, mientras que en los Centros Empresariales Innovadores (CEI), desde su creación (1992) tan sólo se han instalado 43 nuevas empresas, y en la universidad apenas han surgido procesos spin-off, entre otros motivos, por el desconocimiento del profesorado del mercado y por el sistema de evaluación existente.

Por otra parte, la descentralización productiva ha supuesto la especialización de las empresas autóctonas en aquellas partes del proceso que requieran sobre todo un nivel tecnológico de tipo básico y auxiliar, según la terminología de Fernández y Fernández (1988); y la incorporación de los sistemas del kan-ban, just in time, CAD/CAM. dando lugar a la firma de acuerdos de colaboración y a la constitución de redes de subcontratación. Así, las relaciones de cooperación se sitúan en torno al $67 \%$ en la industria aeronáutica y del automóvil, destacando en el ámbito de la producción, asistencia y servicios al cliente y se han incrementado en $56 \%$ las redes de subcontratación, según nuestros datos; mientras que en la industria electrónica e informática los acuerdos de colaboración y los de subcontratación alcanzan un $45 \%$ y $10 \%$ respectivamente. Y por último, en el sector energético se sitúan entre $37 \%$ y $18 \%$.

En el caso de las multinacionales también se ha llegado a acuerdos de colaboración, por ejemplo, entre Hughes Microelectronics y la filial de la General Motors sobre la fabricación de componentes electrónicos para el automóvil. Igualmente, las firmas Alcatel-Fujitsu-Isofotón (electrónica) llegaron a acuerdos comerciales (Jordá Borrell, 1997a).

Pero, los nuevos sistemas de organización de la subcontratación en estos sectores a escala internacional apuntan a que la industria auxiliar fabrique el producto terminado o completo. Por lo tanto, sólo las empresas que están incorporando los sistemas de producción flexible y la diferenciación del producto (tecnología básica y clave) se encontrarán preparadas para adoptar estrategias de calidad e introducirse directamente en el mercado internacional sin tener que hacerlo a través de las grandes empresas. Así, en la industria aeronáutica sólo 14 PYMES realizan aproximadamente $85 \%$ de la subcontratación en Andalucía debido a que el resto de firmas subcontratadas son poco competitivas desde el punto de vista de 
la estrategia de calidad, pero, aún así, esa minoría de empresas que han incorporado tecnología básica y clave permite, en la actualidad, fabricar luera de CASA un porcentaje alto de la estructura del avión.

Por lo tanto, la existencia de relaciones de subcontratación en los sectores de la aeronáutica, automóvil, tecnologías de información, energético de tipo jerárquico y exclusivista con una sola de las grandes cmpresas representativas de las ramas aludidas reduce la capacidad de diversificar los clientes y de alcanzar una salida directa al mercado, lo que puede conducir a cierta vulnerabilidad del sistema productivo local.

De ahí que las firmas que no sean capaces de introducir tecnología básica y clave en sus sistemas de producción flexible deberán establecer acuerdos $o$ asociaciones con otras empresas para aumentar el tamaño, formar redes, evitar duplicaciones en tecnologías comunes e incrementar su especialización a fin de mejorar la competitividad por la vía de la calidad ya que la inexistencia de aspectos como ingeniería del producto, gestión de compras, homologación, gestión de calidad, provoca la imposibilidad de satisfacer las exigencias de otras empresas foráneas de los sectores de la rama de transporte (aeronáutico, automóvil) y de las tecnologías de la información. Y aún así, posiblemente no estarán preparadas para aprovecharse de la demanda de esas grandes empresas que se hayan establecido en Andalucía y/o en España con motivo de la bajada de barreras de entrada a los sectores oligopólicos o monopólicos que se está dando en los territorios de la Unión Europea. Y tampoco podrán competir con aquellas otras PYMES que fabriquen productos sustitutivos en estos sectores.

De este modo, aunque estos sectores tengan todavía en la actualidad, un mercado de carácter oligopólico y las grandes empresas establecidas en Andalucía compitan mundialmente vendiendo tecnología, se encuentran amenazadas por la entrada de nuevos competidores en el mercado nacional y el modelo de internacionalización de la mayoría de las PYMES autóctonas se relaciona con aquellas actividades que ensamblan componentes a nivel local sin salir directamente al mercado internacional. Consecuentemente, una parte considerable de las empresas autóctonas desarrollan sus actividades a escala provincial (28.6\%) y en el ámbito regional $(15.65 \%)$ (Acosta, 1990).

\section{Aproximación al tamaño del aparato organizacional}

Entonces, de acuerdo con lo que venimos exponiendo a lo largo del trabajo parece claro que en el tejido productivo andaluz predominan las empresas con tecnología básica y auxiliar, el modelo de competitividad imperante es el de la reducción de costes y las firmas innovadoras no constituyen 
grupos de sectores conexos, capaces de interrelacionar el tejido empresarial por medio de la cooperación y la creación de externalidades. A estos hechos, cabe añadir que la mayoría de las estructuras puente para conformar el SCTIA se acaban de crear recientemente, cuando la Junta a comienzos de la década modificó la política de creación del sector público, implicando a las grandes empresas y a las multinacionales (Nuintel, Sevillana de Electricidad, Indra, Fujitsu, Abengoa, IBM, Sevillana Gas, Ghesa) en la creación de empresas mixtas de servicios avanzados y estructuras interfase bajo la dirección del Instituto de Fomento Andaluz (IFA). Hasta ahora estas instituciones han empezado por captar instituciones de investigación, centros tecnológicos y empresas con carácter asociado. Sin embargo, apenas se ha implicado a las PYMES debido al bajo nivel de asociacionismo existente.

De ahí que no haya dado lugar a modificar de forma importante la capacidad innovadora del tejido empresarial ni de incrementar en gran medida la integración del SCTIA, dada la juventud de los mismos. No obstante, como la diferenciación de la empresa con su entorno dependerá del desarrollo y el alcance de dichas infraestructuras, de la estructura competitiva de los sectores en Andalucía y de la evolución del mercado único y de la globalización, vamos a intentar medir de forma aproximada el tamaño del aparato organizacional del entorno andaluz. Primeramente, según las necesidades detectadas en las PYMES de algunos sectores innovadores de la estructura industrial andaluza. Y en segundo lugar, de acuerdo con el papel jugado por las estructuras interfase en la creación de recursos locales con poder de integración, interacción y organización (Porter, 1994). El método utilizado consistirá en cruzar las necesidades detectadas en las PYMES con los tipos de servicios ofertados por las estructuras interfase (cuadro 2).

De acuerdo con el informe RITTS (1996) y nuestra encuesta (1996-1997) la pequeña y mediana empresa de los sectores de la agroindustria, electrónica, eléctrica e informática, transportes, química, energía y medio ambiente demandan, en primer lugar, una mejora del sistema de información tecnológico (consulta de patentes, documentos de proyectos ejecutados), de los medios de transferencia de tecnología (redes internacionales, cooperación) y de las fuentes financieras. Por ejemplo: en la industria del transporte las PYMES requerirían una mayor información de la ayuda financiera para la innovación, para crear laboratorios especializados y un mejor conocimiento de los centros de información tecnológica a fin de atenuar la dependencia de las multinacionales y conseguir que el proceso de transferencia tecnológica se realice de forma diversificada (consultoras, universidades, proveedores, centros tecnológicos), y no exclusivamente 
CUADRO 2. Valoración 1 sobre 10 de la oferta y demanda de innovación.

\begin{tabular}{|c|c|c|c|}
\hline Demanda & & Oferta & \\
\hline Mejora sistema ayuda & 7.4 & & \\
\hline Formación empresarial & 7.6 & Formación direct. & 5.7 \\
\hline \multirow[t]{5}{*}{ Mejor inf. soport. } & 7.5 & Asesoram., difus. result. & 8.1 \\
\hline & & Busqueda socios, transf. tec. & $\begin{array}{l}9.1 \\
0.4\end{array}$ \\
\hline & & Servicios información & 0.7 \\
\hline & & Realización inv. aplicada & 3.5 \\
\hline & & $\begin{array}{l}\text { Estudios mercado prospectiva, } \\
\text { tecnológica realiz. foros- } \\
\text { encuentros empresariales } \\
\text { ayuda creac. nuevos empr. }\end{array}$ & 0.7 \\
\hline Aumentar inv. I+TT & 6.4 & Financ., prom. I+D & 10.0 \\
\hline Mejorar imagen exterior & 4.8 & Promoción exterior & 2.5 \\
\hline Laborat. especializado & 4.6 & Medid.,ensay., calibrac. & 4.0 \\
\hline Banca especializada sect. & 3.3 & & \\
\hline $\begin{array}{l}\text { Centros I+TT más } \\
\text { especializados y efect. }\end{array}$ & 3.8 & & \\
\hline Mejorar sist. formac.empleados & 2.7 & Formac direct., etc. & 5.7 \\
\hline Cultura de cluster & 1.5 & & \\
\hline Mecanismos rel. intersectorial & 1.8 & & \\
\hline \multicolumn{4}{|l|}{ Mejorar relación } \\
\hline \multirow[t]{5}{*}{ PYMES-centros 1+TT } & 1.0 & & \\
\hline & & Mejora infraest. básica & 3.5 \\
\hline & & Financiac. bienes equipo & 1.5 \\
\hline & & Financ. global (horizont.) & 1.2 \\
\hline & & Realizar inv. básica & 0.4 \\
\hline
\end{tabular}

Fuente: Elaboración propia a partir del Informe RTTTS (1996). 
con el cliente. Sin embargo, como las estructuras interfase (cuadro 2) han empezado recientemente a ofertar la búsqueda de socios y la transferencia tecnológica no ha habido tiempo suficiente para que este servicio alcanzara un nivel de difusión elevado entre las PYMES a pesar de la importancia relativa $(9 \%)$ que le dan las estructuras puente.

En cuanto a la infraestructura soporte, se detecta que los centros interfase ofrecen, en primer lugar, una diversificación de servicios mucho más amplia que la que demandan las PYMES (cuadro 2), lo que quiere decir y así lo corroboran las memorias de dichos centros que parte de los cursos de formación y en general, de los servicios soporte ofertados son en realidad adquiridos por los organismos públicos, la propia Administración y la gran empresa.

En segundo lugar, se observa que la porción de servicios que demandan las PYMES a las estructuras interfase no están ajustados a sus necesidades, quedando huecos de demanda sin cubrir por motivos diferentes (costes, nivel de preparación exigido o grado de calidad de la divulgación científica). Por ejemplo en el informe RITTS (1996) se hace patente la necesidad de formación en la gerencia de los sectores de agroindustria, transporte, electrónica e informática; igualmente en la industria electrónica-informática persiste la falta de preparación de los técnicos y personal calificado y en general, se da una baja participación de la PYMES en los programas de mejora de calidad a pesar de que ellos son conscientes de la importancia de estos temas para el buen funcionamiento de su empresa. Por lo tanto, a las estructuras interfase y a la Administración les conviene hacer más foros y/o encuentros empresariales sectoriales para descubrir mejor las necesidades de las PYMES, y ajustar con más exactitud la oferta de servicios y las medidas a adoptar, puesto que sólo le dan un valor de $0.7 \%$.

En el caso de la ayuda para la creación de nuevas empresas, estudios de mercado y la mejora de imagen exterior parece, de acuerdo con la información que disponemos (encuesta 1996-1997), que la demanda interrelaciona los tres temas con la internacionalización de la empresa y con el desarrollo de I+DT, mientras las estructuras interfase interpretan la oferta de estos aspectos como departamentos estanco. Pues, con anterioridad al proceso de desarrollo del mercado único existían altas barreras de entrada de tipo monopolístico (energético, químico) en muchos sectores y bajo poder de rivalidad entre los competidores.

Por otra parte, las necesidades de I+DT para cumplir normatividades medioambientales, desarrollar nuevas tecnologías de proceso y de producto eran menores; por lo tanto, el riesgo de introducir productos sustitutivos era más bajo que el actual. Igualmente los requerimientos de personal calificado y de trabajar conjuntamente con grandes clientes 
también eran reducidos. De ahí que a las variables mejora de imagen exterior, formación del empresario y los estudios de mercado, la administración y las estructuras interfase le dieran mucha menos importancia que en la actualidad y no se requería conocer en profundidad las necesidades de las PYMES en materia de I+DT ni sus tendencias.

Hoy, la necesidad de diseñar una política comercial (químico, medioambiental, automoción) ante la entrada en el mercado andaluz de nuevas empresas con alto grado de rivalidad, entendemos que va unido a la adquisición de conocimientos tecnológicos por la vía del desarrollo de I+DT y de la calidad, dado que nos encontramos ante un mercado regional en expansión muy competitivo que requiere crear un mayor número de industrias auxiliares (electrónico, informático, aeronáutico, automoción) con un nivel tecnológico adecuado, ligado a la internacionalización, a fin de que la subcontratación pueda ser de especialización y las PYMES lleguen directamente al mercado.

Por último, la necesidad de ciertos sectores (química, automoción, aeronáutica y agroindustria) de mejorar su imagen exterior e incrementar la internacionalización demuestra que si bien existe una oferta muy amplia para llevar a cabo I+DT, hasta ahora, la mayoría de estructuras interfase han trabajado con las grandes empresas regionales, con la mediana empresa, con los organismos de la administración, con la universidad y con el csiC (Centro superior de Investigaciones Científicas).

Por ello, siendo así que tenemos un número de sectores importante, constituidos por una mayoría de PYMES que necesita fuertes inversiones en 1+DT (agroindustria), sufren procesos de innovación cambiantes (energía, medio ambiente, eléctrico, electrónico, informático, químico), se encuentren amenazados por un alto número de competidores internacionales (químico y medio ambiente) y por la entrada de productos sustitutivos (químico, automoción, energético), de acuerdo con nuestra encuesta, convendría mejorar el nivel de preparación, los canales de financiamiento y los de información (química) para así poder establecer circuitos de transferencia de conocimientos relativos a nuevos procesos y productos entre estos sectores, los centros de investigación aplicada y la universidad.

Por último, tendríamos un grupo de demandas para las que todavía no existe una respuesta por parte de las estructuras interfase (creación de centros de I+TT especializados, difusión de la cultura de cluster, mecanismos de relación intersectorial y la mejora de relaciones entre las PYMES y los centros de $1+\mathrm{TT}$ ) porque el gobierno regional no se ha dado cuenta hasta ahora que los parques tecnológicos y el CESEAND no son instrumentos lo suficientemente potentes para cambiar el entorno regional por sí solos y éstos unicamente podrían efectuar esa labor si estuvieran acompañados de 
una eficaz política de innovación. Pero, como estas demandas constituycı instrumentos favorecedores de la aparición de economías de escala y d aprendizaje tecnológico, el Plan Andaluz de Investigación II (1996), hizo, patentes las medidas necesarias para articular el subsistema productivo con el científico intuyendo que la PYMES constituyen la piedra angular del proceso de innovación.

\section{CONCLUSIONES}

Partimos de la base de que los factores fundamentales del cambio tecnológico andaluz son el mercado único y las instituciones regionales debido a las características de la estructura competitiva de los sectores industriales y al débil nivel de internacionalización de la economía. Esas dificultades han llevado a la Junta de Andalucía a diseñar el modelo innovador de forma escalonada en el tiempo, produciéndose cambios de énfasis significativos, tanto en las prioridades de la articulación y conformación del sistema ciencia-tecnología-industria como en el protagonismo del sector público.

Hasta comienzos de los años noventa, el desarrollo del proceso de innovación consistía esencialmente en la atracción de multinacionales y grandes empresas para crear tejido empresarial y en la aplicación de incentivos a la modernización tecnológica pero sin dar grandes resultados. Fue a partir del Plan de Desarrollo Económico Regional (PADE) 1991-1994 y del Plan Industrial Regional 1994-1997 cuando se aprueba el financiamiento para fomentar $\mathrm{I}+\mathrm{D}$, implantar infraestructura tecnológica y generar entorno mediante la utilización de recursos locales con poder de integración, interacción y organización. Aún así, como la conformación del sistema ciencia-tecnología-industria todavía se encontraba en un estadio de desarrollo embrionario, sobre todo por el bajo nivel tecnológico del tejido industrial, el gobierno regional decidió no poner en marcha una estrategia de innovación industrial y aplicar las medidas adoptadas principalmente al subsistema científico que era el más avanzado.

Sin embargo, las implicaciones del mercado único en la búsqueda de nuevos productos y nuevas formas de organización de la producción condujo a los grandes grupos industriales, establecidos en Andalucía a la incorporación de la automatización y de las nuevas tecnologías de organización flexible, lo que ha supuesto entre 1994 y 1996 un incremento de la inversión de un $57.6 \%$ en nuevos equipos y lo que es más importante, significó la afloración en las grandes empresas de la necesidad de presionar a las PYMES para que incorporen maquinaria automática y flexible, a fin de implantar en éstas la organización del just in time e introducir en ellas 
las nuevas tecnologías que exige este sistema (control de calidad total o círculos de control de calidad, sistemas de control de producción, sistemas de planificación y control de inventarios) contribuyendo a una mejora sustancial de la productividad. En la actualidad, 20\% de las PYMES de los sectores innovadores han incorporado los equipos automáticos siendo de tecnología básica y/o auxiliar, pero únicamente $30 \%$ de ellas han instalado laboratorio y emplean manual y procedimientos de calidad.

Por otro lado, también la entrada en vigor del mercado único llevó al gobierno regional a centrar algunos de sus esfuerzos, de una parte, en la articulación de la industria desde el punto de vista sectorial introduciendo la idea de cluster en ciertas ramas industriales emergentes (por ejemplo: electrónica, metalmecánico). Y de otra, a la modificación de la política de creación del sector público implicando a las grandes empresas y a las multinacionales en la creación de servicios avanzados, estructuras interfase, establecimiento de redes de subcontratación. Pues ésta constituía la mejor forma de introducir al sector privado en la creación de entorno y de favorecer la aparición de economías de escala y de aprendizaje tecnológico. $^{2}$

Así, este proceso de cambio tecnológico provocado, en nuestra opinión por el desarrollo del mercado único y favorecido por las instituciones regionales ha dado lugar en el corto espacio de tiempo transcurrido entre 1990 y 1998 a una modernización tecnológica, a la conformación de la trama organizacional del entorno y a la aparición de algunos clusters innovadores de sectores conexos relacionados con la industria que tiende a externalizar gran parte de su proceso de producción (automóvil, aeronáutica, electrónica) y con aquella otra que ha desarrollado mercados de tipo oligopólico (energía, química básica).

También la renovación de maquinaria y el proceso de externalización de la producción está modificando la organización de ésta última y del trabajo en las empresas, e incluso ha creado nuevas cualificaciones, ha incrementado el nivel tecnológico de las PYMES y se han creado en ellas departamentos de ingeniería. Según datos de nuestra encuesta, del total de entrevistas (20) efectuadas a las estructuras interfase sobre la penetración

\footnotetext{
2 En total existen además de las ocho universidades y los centros del csic, 60 centros (estructuras interfase y de servicios), de los cuales 25 se pueden considerar especializados en los sectores prioritarios (informática, telecomunicaciones, electrónica, energías renovables, productos químicos, medioambiente, biotecnología, metalmecánica y nuevos materiales), en tecnologías horizontales (Agua, medio ambiente) y en servicios trasversales (Centros de Empresas de Innovación [CEI], Centros Empresariales de Servicios Avanzados, Parque Tecnológico de Málaga, Centro de Diseño y Fabricación Asistida por Ordenador (CAD/CAM), Instituto Andaluz de Tecnología).
} 
de los instrumentos de la innovación en el mercado andaluz, $50 \%$ obedeci a la resolución de problemas de ingeniería, $30 \%$ a mejoras de producto y diseño, y $20 \%$ a innovaciones propiamente dichas. Además, de esas dificultades por resolver, $70 \%$ correspondería a la gran empresa y $30 \%$ a las PYMES (energía, agroindustria, metalmecánico, transporte, medio ambiente y nuevas tecnologías de la información e industria farmacéutica). En consecuencia, tanto las funciones desempeñadas (asesoramiento, diagnóstico, ensayos, formación e ingeniería) por las estructuras interfase como las estrategias adoptadas para incrementar el mercado van ligados a las etapas previas que debe seguir una empresa cuando se quiere introducir en el mundo de la innovación (mejora de la gestión empresarial, puesta en marcha de sistemas de calidad, implantación del manual de calidad, mejora de diseño existente, técnicas para mejorar el desarrollo de productos).

En el plano territorial, esas estructuras puente han empezado por captar instituciones de investigación, centros tecnológicos (en algunos casos) y empresas con carácter asociado ${ }^{3}$ de los sectores prioritarios del Plan Andaluz de Desarrollo Económico (PADE). Este inicio de asociacionismo y su implantación en ciertas áreas industriales ha permitido a las estructuras interfase y a los servicios avanzados generar un entramado organizacional mediante la creación de delegaciones en las capitales de provincia, áreas metropolitanas y las ciudades más importantes de Andalucía conforme se detectan cuotas de mercado potenciales. Pero, no constituyen todavía un elemento fundamental para la incorporación de la innovación en Andalucía ni para la realización de transferencia de tecnología a las PYMES.

Por otra parte, también las universidades, centros de investigación, los facilitadores y las empresas innovadoras se localizan en las capitales de provincia y en las áreas metropolitanas. Es decir, el entramado organizacional del entorno al que se ha llegado hasta ahora es el de escala provincial. En las ciudades medias cuando existe industria (sectores tradicionales: calzado, textil, cerámica, orfebrería y la agroindustria) se ubican un número muy reducido de servicios avanzados.

Por último, queremos dejar constancia que la creación de factores avanzados y el desarrollo de la fase de inversión constituyen estadios fundamentales en Andalucía (Jordá Borrell, 1997b) para añadir complejidad y profundidad a los grupos de sectores conexos y de apoyo. Y que en

3 En el conjunto del tejido industrial andaluz hemos podido contabilizar 21 redes de cooperación tecnológica conformadas para la realización de proyectos financiados por el Programa Marco, otras tantas no determinadas para conseguir subvenciones del CDT7 y las 15 creadas en el parque tecnológico de Málaga. 
cl caso andaluz van acompañadas dichas fases, de la introducción de las nuevas tecnologías de organización de la producción, la automatización, la estrategia de calidad y la nueva organización del trabajo, a fin de organizar en su entorno una malla de redes de cooperación de I+DT entre los grandes grupos industriales, las industrias auxiliares, las empresas proveedoras y las firmas clientes capaces de desarrollar economías de escala externas suficientemente potentes para producir un efecto de desparramamiento de las actividades innovadoras y transformar así la economía.

Pero la debilidad del desarrollo de los sectores conexos y de apoyo debido a las altas barreras de entrada que caracterizan a ciertos sectores innovadores (químico, energético, maquinaria, material eléctrico), el reducido número de competidores existentes en algunas ramas industriales (gestión de residuos, energía), el escaso arraigo de la cultura de cluster y de asociacionismo y el hecho de que la descentralización productiva haya tenido lugar en un escaso número de sectores (automóvil, electrónica, aeronáutica) explican en parte la debilidad de la demanda de innovación interior, la escasez de economías de escala externas y en definitiva, la no generación del efecto de desparramamiento.

\section{BIBLIOGRAFIA}

ACOSTA Coleto, A 1990. Informe sobre el sector industrial en Andalucía, Sevilla, IFA, Consejería de Economía y Hacienda.

DOSI,G. 1992. "Fuentes, métodos y efectos microeconómicos de la innovación", en Ekonomiaz, núm. 22, pp. 269-331.

FERNÁNDEZ \& Fernández. 1988. Manual de dirección estratégica de la tecnología, Barcelona, Ariel.

GAFFARD y Quéré.1996. "Innovación, modelos de crecimiento y desarrolo local", en ICE, núm. 754, junio, pp. 25-38.

INSTITUTO ANDALUZ TECNOLOGÍA. 1996. Innovación y transferencia de tecnología (Proyecto RITTS 037), Sevilla, IAT, IFA.

JORDÁ Borrell, R. 1992. Las relaciones de la ciencia-tecnología-industria y el papel de la Administración, Sevilla, Instituto de Desarrollo Regional, Universidad de Sevilla.

- 1997a. "Formación del Sistema-Ciencia-Tecnología-Industria" en Valles, (Coord). 1997. Economía andaluza, Sevilla, Algaida editores, Grupo Anaya

- 1997b. "Nuevas Tecnologías", en Valles, J. (Coord.). 1997. Economía Española, Madrid, McGraw-Hill. 
LUCAS, R. 1993. "Making a Miracle", Econométrica, vol. 61.

MOLERO, J. 1996. "La exportación de tecnología como factor estratégico del desarrollo industrial: un análisis sectorial”, ICE, núm. 752, pp. 105-119.

PORTER, M. 1994. The Competitive Advantage of Nations, China, Macmillan. 